\title{
Performance analysis of direct detection and coherent detection system for optical OFDM using QAM and DPSK
}

\author{
Veneetha Nair ${ }^{1}$, Joseph George K.N. ${ }^{2}$ Asha R.S. ${ }^{3}$ \\ (Department of Electronics Engineering, Govt. Model Engineering College, India)
}

\begin{abstract}
In this paper a theoretical study is conducted regarding the effect of OOFDM in radio over fiber networks (RoF). The system was simulated at 50Gbps for a transmission distance of $1000 \mathrm{~km}$ over standard single mode fiber. A performance analysis was conducted to compare the performance of direct detection and coherent detection schemes in terms of their BER, dispersion tolerance and Q factor. The improvement in performance with the use of polarization diversity schemes in CO-OFDM is studied. The study was conducted with QAM and DPSK modulations. The effect of local oscillator power in coherent detection, and that of the laser launch power are studied. The study also shows the slight degradation in performance with increase in subcarrier.
\end{abstract}

Keywords: - Coherent detection, OOFDM, Q Factor, RoF

\section{INTRODUCTION}

There has been a drastic increase in the demand for high speed communication with mobile connectivity. High speed communication has been traditionally facilitated over wired infrastructure , particularly optical fiber, while mobile communication is supported by wireless infrastructure. But the wireless network has severe performance degradation due to interference, low data rate etc. The design of communication networks that feature high-speed and mobility is facilitated by radio over fiber networks [1].

Orthogonal frequency division multiplexing (OFDM) has been an important candidate in wireless communication since many years. Recently optical OFDM has emerged as a new trend in optical communication networks to combat the effects of dispersion in optical fiber[2][5][6]. Optical OFDM is mainly classified into direct detection system and coherent detection system. In direct detection system a single photodiode is used, While in coherent detection the principle of optical mixing is utilized with local oscillator and optical hybrid. The superior performance of CO-OFDM in terms of its dispersion tolerance and OSNR requirements makes it a suitable candidate for long haul networks.

Coherent reception requires that the polarization of local oscillator should be matched with that of the received signal .Otherwise severe performance degradation results. The state of polarization of light travelling through the conventional fiber varies randomly. Hence polarization tracking device is necessary at the receiver, which is impractical in field application. Another alternative is to split the received light into two orthogonal polarizations and process in separate branches of the receiver. The performance of such a diversity receiver would be independent of the state of polarization of the received signal [3] [4].

The paper presents a performance analysis of a coherent optical OFDM system in terms of its BER. Also a performance comparison is done to show the significance of polarization diversity scheme in coherent architecture. A detailed description of CO- OFDM system is given in section 2. Section 3 details the simulations. The results and discussions are given in section 4 and the paper is concluded in section 5 .

\section{PRINCIPLE OF CO-OFDM}

Orthogonal frequency division multiplexing (OFDM) has been extensively used in wireless communication since many years, due to its excellent immunity to multipath fading effects and frequency selective fading. Recently an optical equivalent for such a system has emerged, known as CO-OFDM. This combines the advantages of coherent detection as well as that of OFDM.

OFDM is a multicarrier modulation technique, where the sub carriers are mutually orthogonal to one another. The main principle of OFDM involves the conversion of a serial data stream into a set of parallel data streams of longer time duration[ 7]. In CO-OFDM transmitter section, the data is first mapped into any constellation like QAM or PSK and is then passed on to an IFFT modulator, to obtain the OFDM spectrum. The easy implementation of OFDM using IFFT modulation, is an important attraction for this architecture. The data is then directly up converted to optical frequency. Thus the electrical bandwidth requirement and thus the cost of transceiver is greatly reduced in CO OFDM. The block diagram of CO-OFDM is shown in Fig 1. 


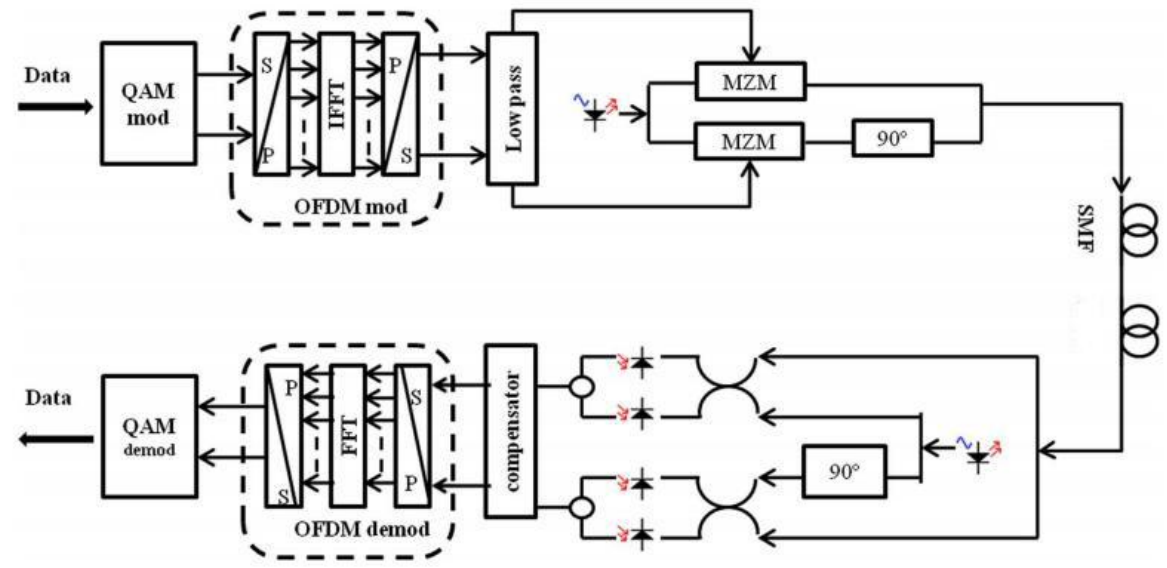

Fig 1: Block diagram of CO-OFDM

The receiver section uses two balanced receivers for photodetection. It is followed by the OFDM demodulator section. Coherent requires that the state of polarization of the incoming light wave be same as that of the LO light wave .Otherwise severe performance degradation results. The SOP of the light wave changes randomly as it propagates through the conventional fiber, due to structural and temperature changes. Hence

either mannual polarization tracking is needed at the receiver, or else the reception has to be made independent of the received polarization, using polarization diversity schemes[4].

\section{SIMULATIONS}

In order to compare analyze the performance of CO-OFDM, a coherent system was simulated with conventional QAM modulation format and a similar system was simulated with CO-OFDM modulation. The simulations were done in versatile optical simulation software.

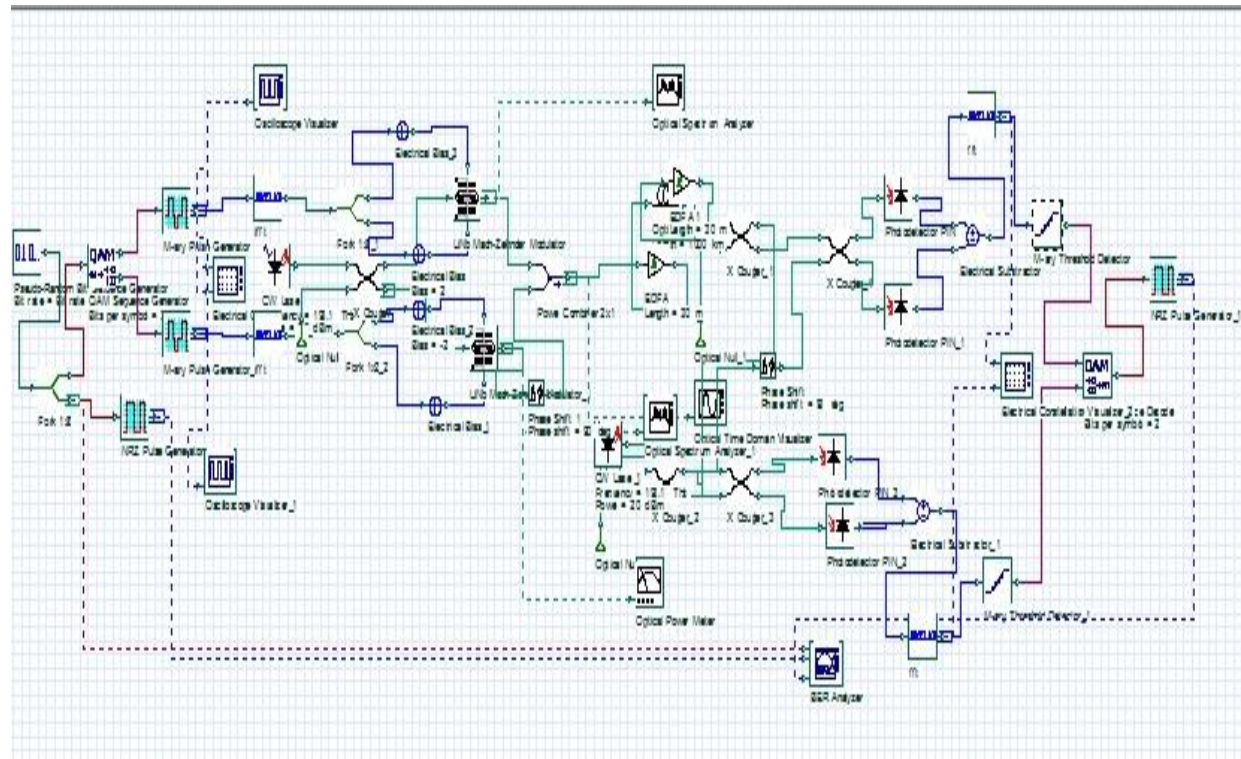

Fig 2: Layout of CO-OFDM system

A 4 QAM coherent optical OFDM system was simulated with 8192 samples at 50 gbps. The number of subcarriers used for OFDM was 4 and no additional cyclic prefix was added. The modulation format used was 4 QAM, and transmission was achieved over a distance of $1000 \mathrm{~km}$ with a $\mathrm{Q}$ factor of 8.62 decibel and a BER of $4.2 \times 10^{-3}$. The performance of above described CO-OOFDM system was compared with that of a coherent detection QAM system for the same transmission distance and data rate

The layout of simulation of CO-OFDM is shown in fig 2, the layout of CO QAM is similar, except the absence of IFFT modulator( hence it is not shown here). The performance of QAM system was inferior to that of OFDM with a BER of $3 \times 10^{-2}$ and a $\mathrm{Q}$ factor of $5 \mathrm{~dB}$. The effect of subcarrier count and the laser launch power in BER of the system was also analyzed with the same layout shown in Fig 2. 
를 Optical Spectrum Analyzer

Dbl Click On Objects to open properties. Move Objects with Mouse Drag

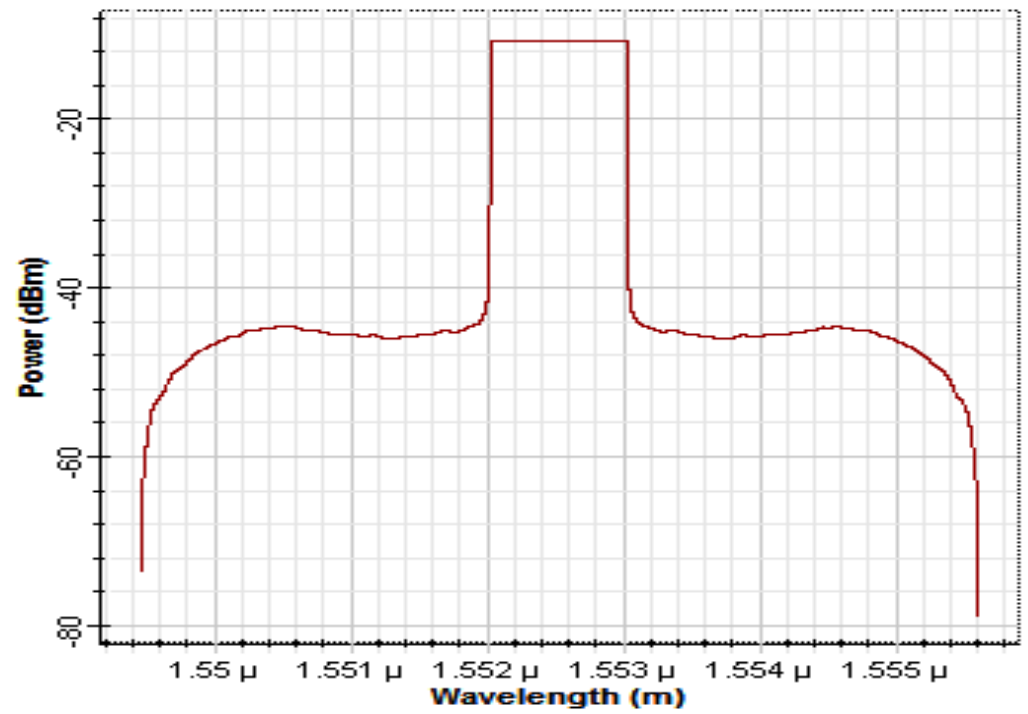

Fig 3: Spectrum of 4 QAM CO-OFDM

In order to improve the performance of coherent OFDM system, the receiver structure is made independent of the incoming polarization by using polarization diversity schemes. As shown in above fig at the transmit end, only one

optical OFDM transmitter is used. However,

compared with the previous system, the two orthogonal polarizations are separately detected in the receiver section. The receiver shown in fig is the so-called polarization diversity receiver. As such, there is no need for optical polarization control. Therefore, the system is resilient to PMD when the polarization-diversity receiver is used.

The above shown layout in Fig 4: was simulated with 8192 samples at 50 gbps and the transmission distance is $1000 \mathrm{~km}$ over standard single mode fiber (SSMF). In order to study the effect of PMD tolerance an additional DGD of 71 ps was added with a PMD emulator. There was no dispersion compensation technique used. The number of subcarriers used is 64 for OFDM. The BER of the system is $3.3 \times 10-5$ and Q factor is $11.36 \mathrm{~dB}$. A similar layout was simulated

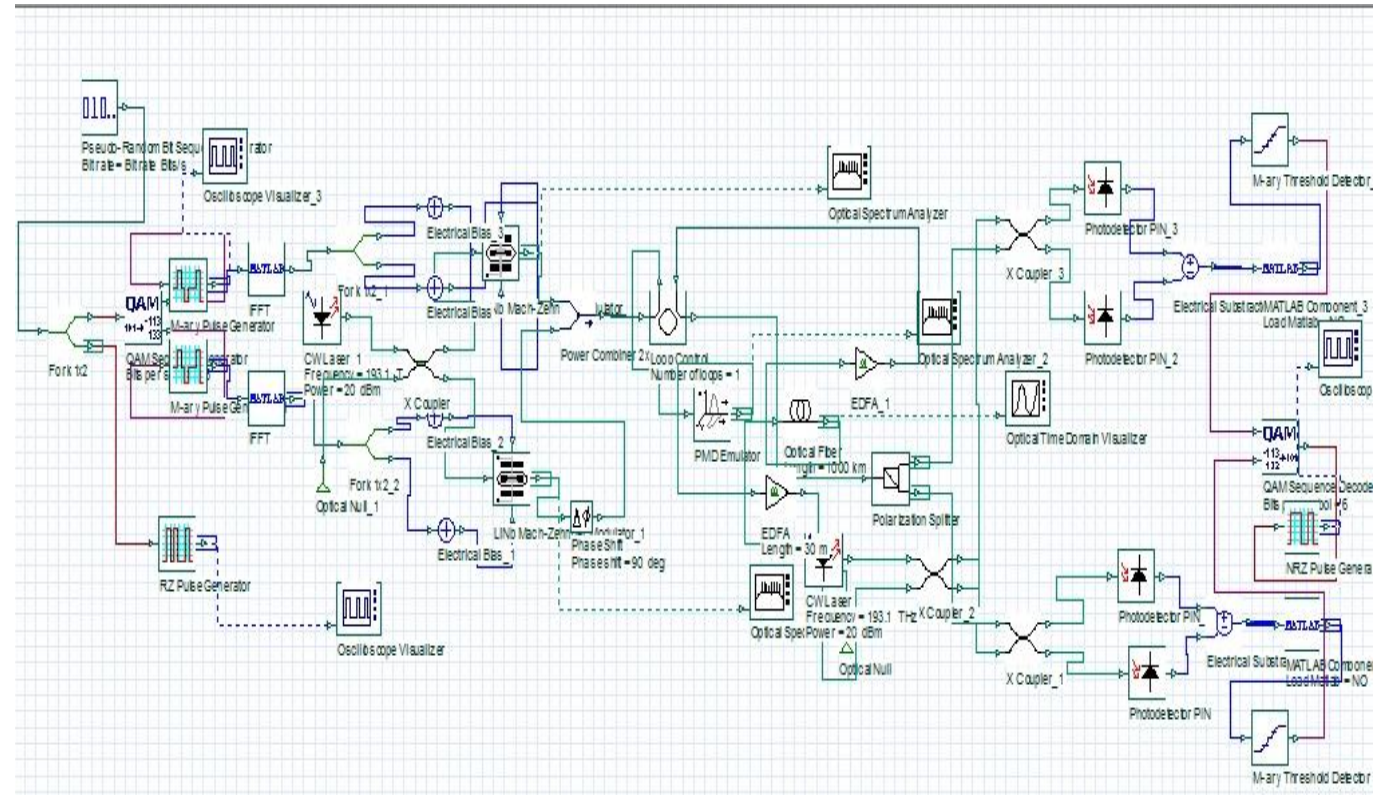

Fig 4: Layout of CO-OFDM with receive diversity 
with 4 QAM modulation, i.e. 4 subcarriers, The BER obtained was 4.6 x 10-6. When compared with 64 QAM COOOFDM it can be observed that the system BER decreases for 4 QAM system. That is as the number of subcarriers increases the BER decreases. The same layout was simulated for higher values of DGD and 300ps was found to be the maximum tolerable DGD.

Another system, employing polarization diversity transmitter was simulated for same data rate and transmission conditions. The BER obtained was 7.1 X 10-4. The Q factor obtained is $10 \mathrm{~dB}$. The performance analysis of an intensity modulation direct detection OOFDM system was also done using DPSK modulation. The performance was observed to be inferior compared to its coherent counterparts. A BER of 9 X $10^{-2}$ was obtained for the system.

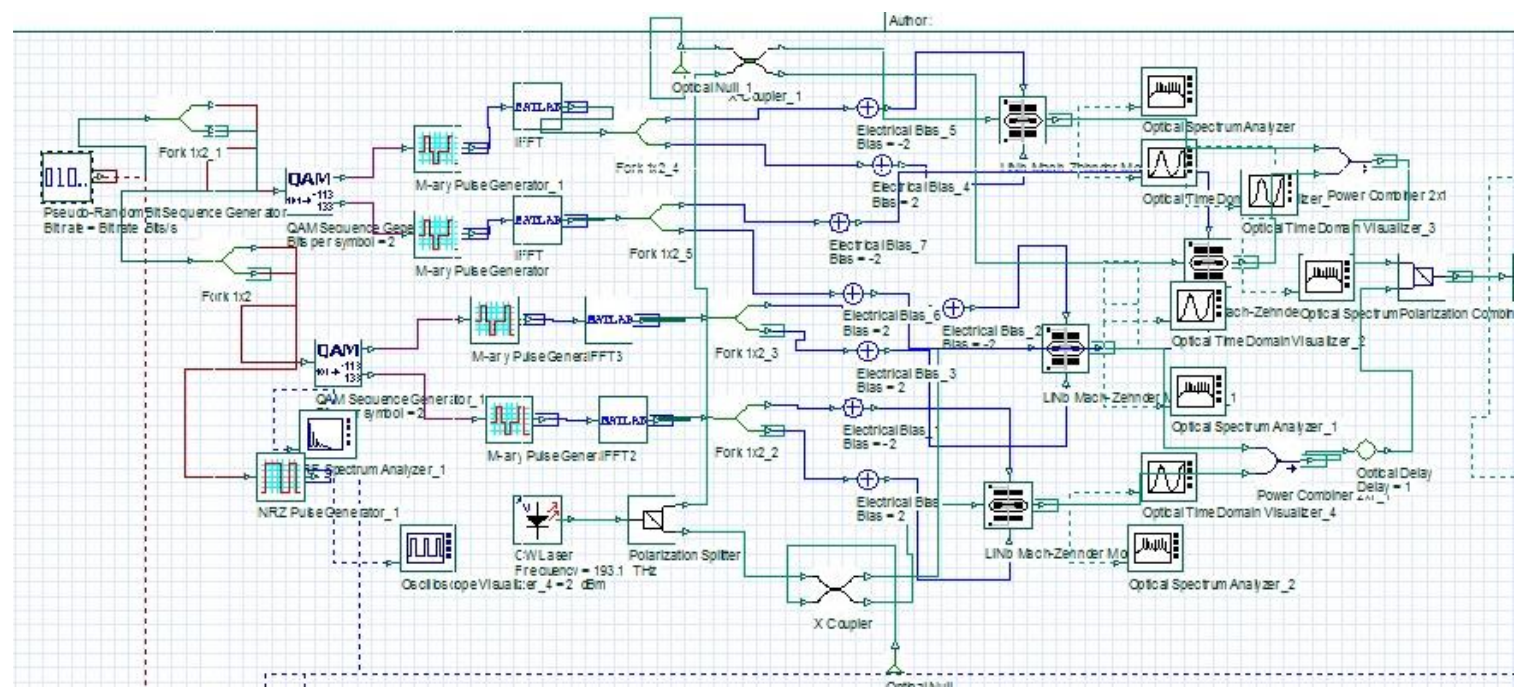

Fig 5: Layout of polarization diversity transmitter section in transmit diversity architecture (4 QAM)

\section{RESULTS AND DISCUSSIONS}

In the above simulations, no dispersion compensation mechanisms were used. It allows the reuse of conventional standard single mode fibers in long haul networks. The use of OOFDM in radio over fiber networks evidently showed superior performance compared to conventional QAM modulation.

The effect of local oscillator power and laser launch power was observed to be have opposite effects on symbol error of the system. While increase in LO power reduced the symbol error, launch power seemed to increase the symbol error. The LO power increases OSNR at the receiver while launch power causes increase in fiber nonlinearity.

Another observation is the increase in BER with increase in subcarrier count. The reason is the increase in carrier offset problems due to increased number of carriers.

\begin{tabular}{|c|c|c|c|c|c|}
\hline & Ifower casm & or Vecrorl & $1090 \mathrm{~T}$ & U ractor & pyomoor error \\
\hline 1 & -10 & 0.107711 & -4.10092 & 3.94468 & $5.92647 \mathrm{e}-005$ \\
\hline 2 & -5 & 0.107711 & -4.10091 & 3.94468 & $7.9267 \mathrm{e}-00 \mathrm{~s}$ \\
\hline 3 & o & 0.107711 & -4.10088 & 3.94468 & $.927126-005$ \\
\hline 4 & 2 & 0.107711 & -4.10087 & 3.94468 & $.927366-005$ \\
\hline 5 & 5 & 0.107711 & -4.10084 & 3.94468 & 92785 e-005 \\
\hline 6 & 10 & 0.107711 & -4.10077 & 3.94468 & $.92915 e-005$ \\
\hline 7 & 15 & 0.107711 & -4.10065 & 3.94468 & $931460-005$ \\
\hline 8 & 20 & 0.107711 & -4.10042 & 3.94468 & -93sse-0os \\
\hline 9 & & & & & \\
\hline
\end{tabular}

Variation of symbol error with laser power (4QAM system)

\begin{tabular}{|c|c|c|c|c|c|}
\hline & Symbol Error] & Error Vector & 100 of & Qfactor & Power (dem) \\
\hline 1 & 0.000202687 & 0.110074 & -3.69317 & 3.71162 & -10 \\
\hline 2 & 0.000202699 & 0.110074 & -3.69315 & 3.71162 & -5 \\
\hline 3 & 0.000202719 & 0.110074 & -3.69311 & 3.71162 & 0 \\
\hline 4 & 0.000202731 & 0.110074 & -3.69308 & 3.71162 & 2 \\
\hline 5 & 0.000202754 & 0.110075 & -3.69303 & 3.71162 & 5 \\
\hline 6 & 0.000202818 & 0.110075 & -3.69289 & 3.71162 & 10 \\
\hline 7 & 0.000202931 & 0.110077 & -3.69265 & 3.71162 & 15 \\
\hline$B$ & 0.000203132 & 0,11008 & -3.69222 & 3.71162 & 20 \\
\hline
\end{tabular}

Variation of symbol error with laser power (64QAM system)

Fig 6: Effect of subcarrier count in OFDM 


\section{Frror at User Defined Decision Instant (Pon}

Dbl Click On Objects to open properties. Move Objects with Mous

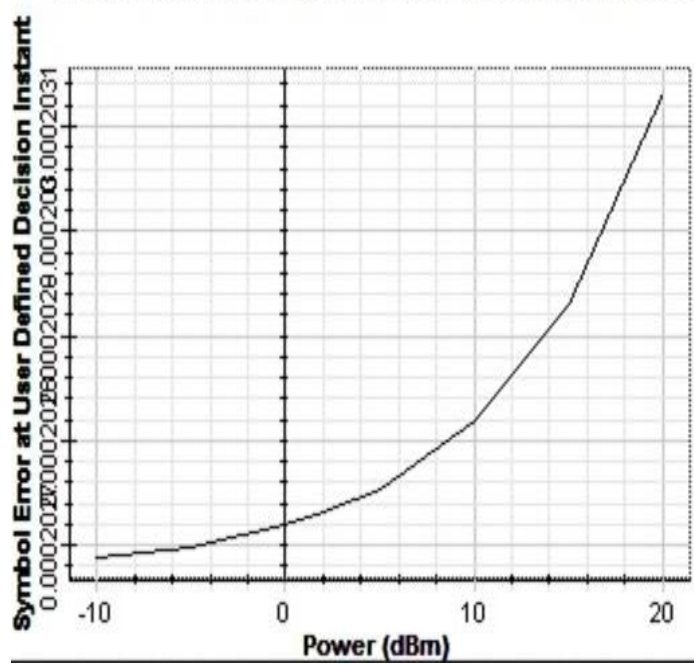

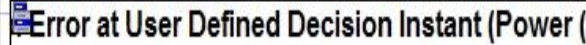

Dbl Click On Objects to open properties. Move Objects with Mouse Dre

萡

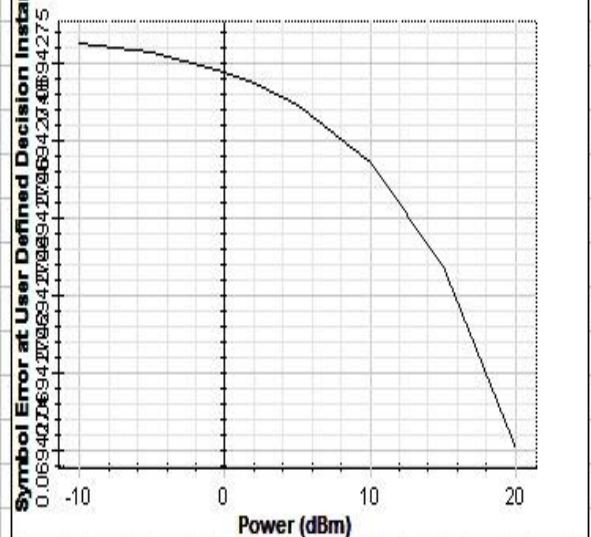

Fig 7: (a) Variation of symbol error with laser launch power (b) variation of symbol error with local oscillator power

The simulation results shows the superior performance of OOFDM system over other conventional transmission system. The use of polarization diversity scheme further improve the performance of COOFDM. The comparison of performance of different architectures simulated are summarized in Table 1.

Table 1: Comparison of performance

\begin{tabular}{|c|c|c|c|c|c|c|}
\hline Architecture & $\begin{array}{l}\text { Modulation } \\
\text { format }\end{array}$ & $\begin{array}{l}\text { Launch power } \\
\text { (dbm) }\end{array}$ & Symbol error & BER & $\begin{array}{l}\text { Q Factor } \\
\text { (db) }\end{array}$ & PMD Resilance \\
\hline Direct detection & DPSK & 20 & .068 & $1.1 \times 10^{\circ}-2$ & $<3$ & no \\
\hline $\begin{array}{l}\text { Simple } \\
\text { COOFDM }\end{array}$ & 4QAM & 0 & .008 & $4.2 \times 10^{n}-3$ & 8.39 & No \\
\hline $\begin{array}{l}\text { COOFDM- } \\
\text { receive diversity }\end{array}$ & 64 QAM & 20 & .000202 & $3.3 \times 10^{-5}$ & 11.36 & Yes \\
\hline $\begin{array}{l}\text { COOFDM- } \\
\text { transmit|diversity }\end{array}$ & 4QAM & 0 & .00142 & $7 \times 10^{\wedge} 4$ & 10.04 & Yes \\
\hline
\end{tabular}

V. CONCLUSION

The simulations were done at $50 \mathrm{gbps}$ and a transmission distance of $1000 \mathrm{~km}$ was studied through SSMF. The use of COOFDM allows the use of SSMF in long haul networks without any dispersion compensation technique. The dispersion tolerance of the OOFDM system is superior compared to conventional modulation techniques.

The coherent detection OFDM exhibits a $\mathrm{Q}$ factor improvement of about $9 \mathrm{db}$ compared to direct detection. The use of polarization diversity schemes further improve the performance of CO-OFDM. The use of Polarization diversity schemes showed excellent PMD tolerance of about 300ps and still maintaining a satisfactory BER performance of about $10^{-5}$. The receive diversity architecture exhibits best BER performance compared to other transmit diversity scheme. 


\section{REFERENCE}

[1]. Siddharth Ray, Muriel M'edard and Lizhong Zheng, Fiber Aided Wireless Network Architecture IEEE Journal On Selected Areas In Communications, Vol. 29, No. 6, June 2011

[2]. Qi Yang, Yan Tang, Yiran Ma, and William Shieh, Experimental Demonstration and Numerical Simulation of 107-Gb/s High Spectral Efficiency Coherent Optical OFDM Journal Of Lightwave Technology, Vol. 27, No. 3, February 1, 2009.

[3]. John R Barry and Edward A Lee, Performance of coherent optical receivers Proceedings of the IEEE, Vol 78, No 8 August 1990

[4]. W. Shieh, X. Yi, Y. Ma, and Y. Tang, Theoretical and experimental study on PMD supported

[5]. transmission using polarization diversity in coherent optical OFDM systems OPTICS EXPRESS 6 August 2007 / Vol. 15, No. 16 / 9936

[6]. Nadia Nowshin, , A K M Arifuzzman, , Mohammed Tarique, Demonstration and performance analysis

[7]. of rof based ofdm-pon system for nextgeneration Fiber optic communication, International Journal of Computer Networks \& Communications (IJCNC) Vol.4, No.1, January 2012

[8]. Yoon-Khang Wong, S. M. Idrus, and I. A. Ghani, Performance Analysis of the OFDM Scheme for Wireless over Fiber Communication Link, International Journal of Computer Theory and Engineering, Vol. 4, No. 5, October 2012

[9]. Ivan B. Djordjevic and Bane Vasic, Orthogonal frequency division multiplexing for

[10]. high-speed optical transmission,2006 OSA 1 May 2006 / Vol. 14, No. 9 / OPTICS EXPRESS 3767 